\title{
Deep vein thrombosis in bilateral lower extremities after hip fracture: a retrospective study of 463 patients
}

This article was published in the following Dove Press journal:

Clinical Interventions in Aging

\author{
Bin-Fei Zhang* \\ Xing Wei* \\ Hai Huang \\ Peng-Fei Wang \\ Ping Liu \\ Shuang-Wei Qu \\ Jia-Hao Li \\ Hu Wang \\ Yu-Xuan Cong \\ Yan Zhuang \\ Kun Zhang
}

Department of Orthopedic Trauma, Hong-Hui Hospital, Xi'an Jiaotong

University College of Medicine, Beilin

District, Xi'an, Shaanxi, China

*These authors contributed equally to this work
Correspondence: Yan Zhuang

555 Youyi East Road, Xi'an, Shaanxi 710054, The People's Republic of China Tel +8602962818342

Email zhuangyan25I2@I26.com
Objective: To investigate the incidences of deep vein thrombosis (DVT) before and after operation in inpatients with hip fractures in both lower extremities.

Patients and methods: We collected the clinical data of 463 patients with lower extremities fractures who presented at Xi' an Honghui Hospital between July 1, 2014, and October 31, 2016 and met all the inclusion criteria. Doppler ultrasonography was used to diagnose DVT. The patients were examined preoperatively and postoperatively and divided into the thrombosis and non-thrombosis group according to the ultrasonographic findings. We divided the DVT cases into central, peripheral, and mixed thromboses.

Results: The incidence of preoperative DVT was $34.98 \%$, and the prevalence of DVT on the uninjured side was $13.60 \%$. This composition ratio increased to $57.23 \%$ postoperatively, and the prevalence of DVT on the uninjured side was $25.05 \%$. Age (odds ratio [OR], 1.03; 95\% CI: $1.01-1.04 ; P=0.002)$, venous thrombosis at admission (OR, $4.05 ; 95 \% \mathrm{CI}$, $2.30-7.13 ; P=0.000)$, and the days between the fracture and the operation $(\mathrm{OR}, 1.10 ; 95 \%$ CI, 1.02-1.20; $P=0.020)$ were the independent risk factors of preoperative DVT. Coronary heart disease (OR, 1.85; 95\% CI: $1.18-2.89 ; P=0.007)$, venous thrombosis at admission (OR, 22.35; 95\% CI: $6.78-73.60 ; P=0.000)$, days between fracture and operation $(\mathrm{OR}, 1.06 ; 95 \%$ CI: $1.01-1.11 ; P=0.021)$, and blood loss (OR, 1.002; 95\% CI: $1.000-1.003 ; P=0.014)$ were independent risk factors of postoperative DVT.

Conclusion: The actual incidence of DVT after hip fracture may be underestimated. The incidences of preoperative and postoperative DVTs and the incidence of DVT on the uninjured limb were high.

Keywords: deep vein thrombosis, ultrasonography, hip fracture, anticoagulation, low-molecularweight heparin

\section{Introduction}

Deep vein thrombosis (DVT) of the lower extremities is a common complication in patients with trauma. It should be prevented and treated without delay; otherwise, it can lead to chronic pain; secondary varicose veins; and ulcers, which can seriously affect the quality of life of patients and even cause fatal pulmonary embolism. ${ }^{1}$ In recent years, orthopedists have paid increasing attention to the prophylaxis and treatment of DVT. At present, drug prevention is considered as one of the most effective methods to decrease the risk of lower extremity DVT. ${ }^{2}$ Traditional low-molecular-weight heparin $(\mathrm{LMWH})^{3}$ is used as the standard protocol.

The prevalence of DVT of the hip after trauma is reported to range from $11.1 \%$ to $32.8 \%{ }^{4-6}$ in recent studies. Pooling data from the injured limbs, Song et al reported 
that $29.4 \%$ of preoperative patients have DVT, and the postoperative incidence has increased to $32.8 \% .{ }^{5}$ In fact, the uninjured limb could also carry a risk of DVT. Meissner et al suggested that the hypercoagulation state persists for at least 1 month after an injury in $80 \%$ of patients. ${ }^{7}$ Therefore, DVT could also arise in the uninjured limb. Decker et al demonstrated that DVT could occur in both the injured and uninjured leg, with a trend for higher incidences in the injured leg. ${ }^{8}$ Mok et al reported that the incidence of DVT in uninjured limbs was $14.3 \% .^{9}$ In addition, some studies reported only symptomatic DVT, ignoring asymptomatic DVT. In fact, Sun et al reported that asymptomatic DVT accounts for about $75 \%$ of all DVTs. ${ }^{10}$ Some studies even reported that all patients who had DVT were clinically asymptomatic. ${ }^{11,12}$

On the basis of these factors, we consider that the actual incidence of DVT after hip fracture is underestimated. This study was aimed at retrospectively analyzing the data of patients with hip fractures at Xi'an Honghui Hospital. The purpose was to investigate the incidences of DVT before and after operation in inpatients with hip fractures in both lower extremities.

\section{Patients and methods Ethical statement}

This study retrospectively analyzed the data of patients with hip fractures who were admitted between July 1, 2014, and October 31, 2016, in Xi' an Honghui Hospital. The study was approved by the institutional review board of Xi'an JiaoTong University, and a signed informed consent form was obtained from each participant.

\section{Inclusion and exclusion criteria}

The inclusion criteria were as follows: 1 ) age $\geq 16$ years; 2) fresh hip fractures that require surgical treatment; 3 ) hospital stay of $>48 \mathrm{~h}$; 4) willing to undergo anticoagulant therapy to prevent thrombosis and submitted a signed informed consent form; and 5) availability of preoperative and postoperative ultrasonography results. The Risk Assessment Profile for Thromboembolism (RAPT) score of the patients was moderate to high.

The exclusion criteria were as follows: hip fractures with delayed treatment ( $>3$ weeks from injury to surgery); open soft tissue fractures; serious medical problems that make the patients intolerant to surgery; poor compliance patients; and patients who refuse to participate in the study. We excluded the delayed hip fractures because of the long time gap between the fracture and the operation. Open soft tissue fractures were also excluded because these injuries were usually related to high energy damage. Also, to prevent allergy, ischemic stroke and bleeding events, patients with anticoagulation contraindications were excluded from the study such as patients with history of allergy to the anticoagulation drugs, patients with acute bacterial endocarditis, thrombocytopenia, fresh hemorrhage, and stroke in 3 months.

\section{Methods}

All the patients who were admitted to the hospital were routinely assessed using the RAPT score for thromboembolism risk. At the same time, we also assessed whether the patients had anticoagulation contraindications. For patients without anticoagulation contraindications, LMWH $(0.4 \mathrm{~mL}$, once a day; Glaxo Wellcome Production, GlaxoSmithKline, Tianjin, China) was subcutaneously injected to prevent DVT. In addition, mechanical thrombo-prophylaxis (pressure pump, 20 min twice a day) was used. Blood samples were collected on admission ( $2 \mathrm{~h}$ after admission), 1 day before the operation, and 1 and 5 days after the operation. The aim testing index included D-dimer and routine blood tests. These timing points were identified to evaluate the patients' general condition and the coagulation state at admission, preoperatively and postoperatively, and before discharge.

We used Doppler ultrasonography to diagnose DVT. The diagnostic criteria were in accordance with the Robinov group's criteria. ${ }^{13}$ The patients were examined before and after operation. All the patients received ultrasonography in both lower limbs 1 day before the planned surgery and on days 3-5 after operation. DVTs were classified into 3 types: central (femoral and iliac veins), peripheral (calf muscle veins, fibular vein, and anterior/posterior tibial vein), and both central and peripheral thrombosis (called mixed DVT; popliteal vein).

According to ultrasonography results, the patients were divided into 2 groups, a thrombosis group and a non-thrombosis group. For the patients without thrombosis, LMWH was continuously subcutaneously injected to prevent DVT. For the patients with thrombosis, LMWH was subcutaneously injected to treat DVT. The protocol of therapeutic anticoagulation was guided by hospital consultation from the department of vascular surgery. When ultrasonography results showed central or mixed thrombosis preoperatively, evaluation was performed by the department of vascular surgery and an inferior vena cava (IVC) filter was used to prevent fatal pulmonary embolism if needed. The anticoagulant therapy was discontinued $12 \mathrm{~h}$ before the surgery 
and resumed $24 \mathrm{~h}$ after the surgery. When the patients were discharged, the protocol was changed to rivaroxaban (prophylaxis dose $10 \mathrm{mg}$ once daily and therapeutic dose $20 \mathrm{mg}$ once daily; Bayer Pharma AG, Germany) until 35 days after the operation.

\section{Statistical analysis}

Statistical analysis was performed using the SPSS (Version 19.0, SPSS Inc., Chicago, IL, USA). The measurement data were assessed for normal or non-normal distribution. An independent sample $t$-test was used in the statistical analysis. For the enumeration data, the chi-square test was used. A multivariate analysis was used to detect the risk factors. The difference was statistically significant $(P<0.05)$.

\section{Results}

\section{Patient characteristics}

In total, 1,684 patients with lower extremity fractures were screened. After applying the exclusion criteria, 463 patients with hip fractures who were admitted to our hospital between July 1, 2014, and October 31, 2016, were included. All the patients were treated with LMWH at admission. The patients' mean $( \pm \mathrm{SD})$ age was $72.86( \pm 13.79)$ years (range, 19-102 years). Of these patients, $288(62.20 \%)$ were female and $175(37.79 \%)$ were male. Of these patients, 218 had intertrochanteric fractures, 17 had subtrochanteric fractures, and 228 had femoral neck fractures. All the patients underwent surgery. Open reduction internal fixations (ORIFs) were performed in 278 patients; hemiarthroplasty in 156 patients; total hip replacement in 28 patients; and external fixation in 1 patient. Of the 463 patients, there were only 2 patients who underwent placement of the IVC filter before operation, and the IVCs were taken out after the operation. Postoperative infection rate was $0.2 \%(1 / 463)$, only 1 woman suffered with superficial infection and it was controlled with an oral antibiotic. No fatal bleeding events or bleeding into a critical site was found in this study.

\section{Prevalence of DVT on preoperative ultrasonography}

On the basis of the preoperative ultrasonography result, the characteristics of the patients with hip fractures, with and without thrombosis, were compared. As a result, 162 patients were found to have thrombosis $(34.98 \%)$ and 301 had no thrombosis (65.01\%; Table 1). Of the cases, 30.9\% were peripheral DVTs; $0.9 \%$ were central DVTs; and 3.2\% were mixed DVTs. Peripheral DVTs constituted 88.27\% (143/162) of all DVTs.
A total of 162 patients had DVT. However, some DVTs existed in the uninjured side and not on the affected side. Of the patients, 20 who had left hip fracture had DVT in both lower extremities, 14 who had left hip fracture had right lower extremity DVT, 16 who had right hip fracture had left lower extremity DVT, and 13 who had right hip fracture had DVT in both lower extremities. Ninety-nine patients had single injured lower extremity DVT. The prevalence of DVT on the uninjured side was $13.60 \%$ (63/463), accounting for $38.89 \%$ $(63 / 162)$ of all preoperative DVTs.

\section{Univariate analysis of preoperative DVT}

Between the 2 groups, no statistically significant differences were observed in sex, unilateral or bilateral limb, type of fracture, medical morbidity (excluding venous thrombosis at admission), body mass index (BMI), American Society of Anesthesiologists classification, D-dimer level at admission and preoperatively, and C-reactive protein (CRP) level at admission. However, the thrombosis group had more patients with an identified venous thrombosis at admission than the non-thrombosis group $(24.69 \%$ vs $8.64 \%, P=0.000)$. In addition, the number of days between the fracture and the hospitalization ( $2.49 \pm 5.12$ vs $1.39 \pm 3.35$ days, $P=0.015)$ and the number of days between the fracture and the operation ( $7.07 \pm 6.12$ vs $5.30 \pm 5.16$ days, $P=0.002$ ) were 2 other vital contributing factors to thrombosis, as shown in Table 1.

\section{Multivariate analysis of preoperative DVT}

A multivariate analysis was used to detect the risk factors by entering age, venous thrombosis at admission, number of days between the fracture and the hospitalization, and number of days between the fracture and the operation. The results showed that age (OR, 1.03; 95\% CI: 1.01-1.04; $P=0.002)$, venous thrombosis at admission (OR, 4.05; 95\% CI: $2.30-7.13 ; P=0.000)$, and the number of days between the fracture and the operation (OR, 1.10; 95\% CI: 1.02-1.20; $P=0.020)$ were independent risk factors of preoperative DVT (Figure 1A).

\section{Prevalence of DVT on postoperative ultrasonography}

All the cases were examined using ultrasonography after the operation to identify the thrombosis in the lower limbs, including 265 patients with thrombosis (57.23\%) and 198 patients without thrombosis $(42.76 \%)$. Of the cases, $49.9 \%$ were peripheral DVT, $1.1 \%$ were central DVT, and $6.3 \%$ were mixed DVT. Peripheral DVTs constituted $87.17 \%$ (231/265) of all DVTs (Table 2). 
Table I Patient characteristics according to preoperative ultrasound

\begin{tabular}{|c|c|c|c|c|}
\hline Items & Thrombosis & No thrombosis & Overall & $P$-value \\
\hline Number & 162 & 301 & 463 & \\
\hline Age & $75.36 \pm 11.93$ & $71.51 \pm 14.53$ & $72.86 \pm 13.79$ & 0.002 \\
\hline \multicolumn{5}{|l|}{ Sex } \\
\hline Female & 109 & 179 & 288 & 0.098 \\
\hline Male & 53 & 122 & 175 & \\
\hline \multicolumn{5}{|l|}{ Unilateral or bilateral fracture } \\
\hline Left low limb & 89 & 169 & 258 & 0.803 \\
\hline Right low limb & 73 & 132 & 205 & \\
\hline \multicolumn{5}{|l|}{ Types of fracture } \\
\hline Intertrochanteric fracture & 82 & 136 & 218 & 0.400 \\
\hline Subtrochanteric fracture & 7 & 10 & 17 & \\
\hline Femoral neck fracture & 73 & 155 & 228 & \\
\hline \multicolumn{5}{|l|}{ Medical morbidity } \\
\hline Hypertension (\%) & $55(33.95)$ & 99 (32.89) & 154 & 0.817 \\
\hline Diabetes $(\%)$ & $26(16.05)$ & $34(\mathrm{I} I .30)$ & 60 & 0.146 \\
\hline Coronary heart disease (\%) & $6 \mathrm{I}(37.65)$ & $94(31.23)$ & 155 & 0.162 \\
\hline Arrhythmia (\%) & $22(13.58)$ & $38(12.62)$ & 60 & 0.770 \\
\hline Stroke $(\%)$ & $13(8.02)$ & $29(9.63)$ & 42 & 0.565 \\
\hline Venous thrombosis at admission (\%) & $40(24.69)$ & $26(8.64)$ & 66 & 0.000 \\
\hline Associated trauma $(\%)$ & $9(5.55)$ & $15(4.98)$ & 24 & 0.791 \\
\hline BMI & $22.45 \pm 3.85$ & $22.49 \pm 3.90$ & $22.48 \pm 3.87$ & 0.948 \\
\hline Days between fracture and hospitalization (days) & $2.49 \pm 5.12$ & $1.39 \pm 3.35$ & $1.77 \pm 4.09$ & 0.015 \\
\hline Days between fracture and operation (days) & $7.07 \pm 6.12$ & $5.30 \pm 5.16$ & $5.93 \pm 5.58$ & 0.002 \\
\hline \multicolumn{5}{|l|}{ ASA classification } \\
\hline I & 5 & 23 & 28 & 0.254 \\
\hline 2 & 107 & 188 & 295 & \\
\hline 3 & 49 & 87 & 136 & \\
\hline 4 & 3 & 1 & 4 & \\
\hline \multicolumn{5}{|l|}{ Serum markers } \\
\hline D-dimer at admission (mg/L) & $14.46 \pm 14.90$ & $|5.72 \pm| 8.7 \mid$ & $15.29 \pm 17.54$ & 0.445 \\
\hline D-dimer preoperatively (mg/L) & $7.46 \pm 7.28$ & $5.80 \pm 9.42$ & $6.50 \pm 8.61$ & 0.153 \\
\hline CRP at admission (mg/L) & $22.47 \pm 34.03$ & $18.39 \pm 29.98$ & $19.75 \pm 31.39$ & 0.297 \\
\hline
\end{tabular}

Notes: Coronary heart disease includes chronic myocardial ischemia, ST segment changes without clinical symptoms, and delayed myocardial infarction in electrocardiograms. Arrhythmia includes all kinds of arrhythmia in electrocardiograms. Stroke includes fresh and delayed cerebral ischemia and hemorrhage on CT or MRI.

Abbreviations: ASA, American Society of Anesthesiologists; BMI, body mass index; CRP, C-reactive protein; CT, computed tomography.

However, some DVTs occurred on the uninjured side and not the affected side. Of the patients, 44 who had left hip fracture had DVT in both lower extremities, 13 who had left hip fracture had right lower extremity DVT, 13 who had right hip fracture had left lower extremity DVT, and 46 who had right hip fracture had DVT in both lower extremities. Single injured lower extremity DVT was found in 149 patients. The prevalence of DVT on the uninjured side was $25.05 \%$ (116/463), accounting for $43.77 \%$ (116/265) of all postoperative DVTs.

\section{Univariate analysis of postoperative DVT}

Between the 2 groups, no statistically significant differences were found in age, unilateral or bilateral limbs, type of fracture, medical morbidity (excluding coronary heart disease or venous thrombosis at admission), BMI, length of hospital stay, number of days between the fracture and the hospitalization, operation duration, transfusion, liquid transfusion, drainage, D-dimer level at admission, and CRP level at admission. However, the thrombosis group had more female patients $(P=0.018)$ than the non-thrombosis group. Similar to the preoperative ultrasonography finding, venous thrombosis at admission was a contributing factor to postoperative thrombosis $(P=0.000)$. In addition, coronary heart disease was another important contributing factor to postoperative thrombosis $(P=0.041)$. The number of days between fracture and operation $(6.49 \pm 5.45$ vs $5.16 \pm 5.70$ days, $P=0.013$ ) was another contributing factor to postoperative thrombosis. Among the serum markers, the D-dimer levels preoperatively, postoperative day 1 , and postoperative day 5 , and the CRP level on postoperative day 5 were obviously higher in the thrombosis group, as shown in Table 2. 


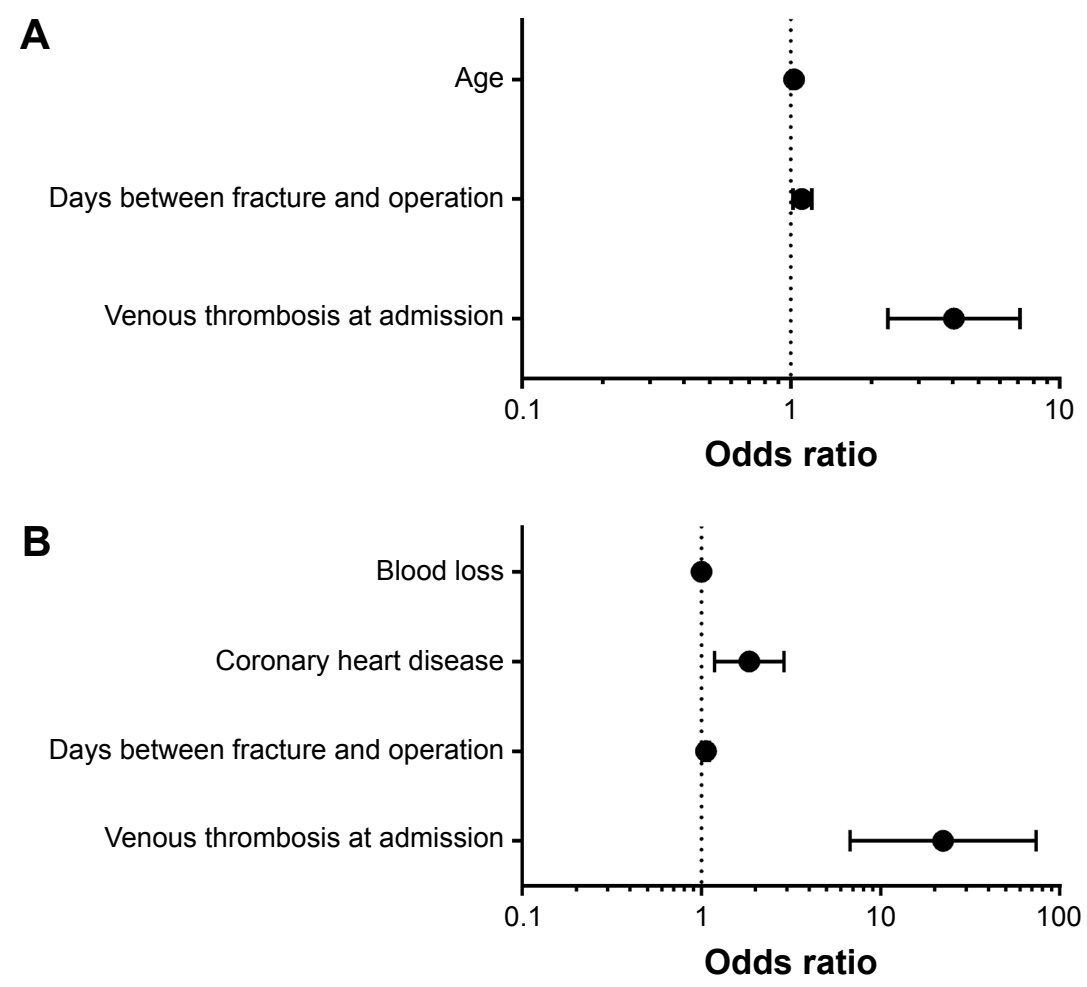

Figure I The risk factors of DVT formation in hip fracture. (A) The contributing factors to preoperative DVT. (B) The contributing factors to postoperative DVT. Abbreviation: DVT, deep vein thrombosis.

\section{Multivariate analysis of postoperative DVT} A multivariate analysis was used to identify the risk factors by entering sex, coronary heart disease, venous thrombosis at admission, number of days between the fracture and the operation, and blood loss. The results showed that coronary heart disease (OR, 1.85; 95\% CI: 1.18-2.89; $P=0.007$ ), venous thrombosis at admission (OR, 22.35; 95\% CI: 6.78-73.60; $P=0.000)$, number of days between fracture and operation (OR, 1.06; 95\% CI: 1.01-1.11; $P=0.021)$, and blood loss (OR, 1.002; 95\% CI: 1.000-1.003; $P=0.014)$ were independent risk factors of postoperative DVT (Figure 1B).

\section{Dynamic changes of DVT before and after operation}

The dynamic changes of DVT before and after operation are shown in Figure 2. We defined "thrombosis" as " 1 " and "no thrombosis" as " 0 " to compare the changes of DVT from preoperatively to postoperatively. The results showed that the postoperative incidence of DVT was higher than its preoperative incidence $(t=9.81, P=0.000)$.

Of the patients, $65.44 \%(39.52 \%, 24.62 \%, 0.22 \%$, and $1.08 \%$ ) showed no changes in hospitalization duration. Moreover, $3.24 \%$ of the patients who had preoperative peripheral DVT no longer had thrombosis postoperatively. In addition,
$25.48 \%(23.11 \%, 0.43 \%$, and $1.94 \%)$ of the patients progressed from having no thrombosis preoperatively to having peripheral, central, or mixed DVT postoperatively.

\section{Discussion}

Most previous research studies mainly analyzed the distribution of DVTs in different fractures ${ }^{3}$ or the contributing risk factors to duration of hospitalization due to DVT. ${ }^{4}$ As far as we know, this is the first retrospective study focusing on the changes of preoperative and postoperative DVTs in patients with hip fractures in both lower limbs, and our findings are as follows: 1) the incidence of preoperative DVT was $34.98 \%$, and this composition ratio increased to $57.23 \%$ postoperatively; 2) the prevalence of preoperative DVT on the uninjured side was $13.60 \%$, accounting for $38.89 \%$ of all preoperative DVTs; the prevalence of postoperative DVT on the uninjured side was $25.05 \%$, accounting for $43.77 \%$ of all postoperative DVTs; 3 ) age, venous thrombosis at admission, and number of days between the fracture and the operation were independent risk factors of preoperative DVT; 4) coronary heart disease, venous thrombosis at admission, number of days between the fracture and the operation, and blood loss were independent risk factors of postoperative DVT; 5) peripheral DVT constituted $88.27 \%$ 
Table 2 Patient characteristics according to postoperative ultrasound

\begin{tabular}{|c|c|c|c|c|}
\hline & Thrombosis & No thrombosis & Overall & $P$-value \\
\hline Number & 265 & 198 & 463 & \\
\hline Age & $73.72 \pm 12.18$ & $71.72 \pm 15.65$ & $72.86 \pm 13.79$ & 0.137 \\
\hline \multicolumn{5}{|l|}{ Sex } \\
\hline Female & 177 & 111 & 288 & \multirow[t]{2}{*}{0.018} \\
\hline Male & 88 & 87 & 175 & \\
\hline \multicolumn{5}{|l|}{ Unilateral or bilateral fracture } \\
\hline Left low limb & 147 & 111 & 258 & \multirow[t]{2}{*}{0.900} \\
\hline Right low limb & 118 & 87 & 205 & \\
\hline \multicolumn{5}{|l|}{ Types of fracture } \\
\hline Intertrochanteric fracture & 128 & 90 & 218 & \multirow[t]{3}{*}{0.805} \\
\hline Subtrochanteric fracture & 9 & 8 & 17 & \\
\hline Femoral neck fracture & 128 & 100 & 228 & \\
\hline \multicolumn{5}{|l|}{ Medical morbidity } \\
\hline Hypertension (\%) & 91 & 63 & 154 & 0.569 \\
\hline Diabetes (\%) & 37 & 23 & 60 & 0.457 \\
\hline Coronary heart disease (\%) & 99 & 56 & 155 & 0.041 \\
\hline Arrhythmia (\%) & 32 & 28 & 60 & 0.513 \\
\hline Stroke (\%) & 20 & 22 & 42 & 0.186 \\
\hline Venous thrombosis at admission (\%) & 63 & 3 & 66 & 0.000 \\
\hline Associated trauma (\%) & 16 & 8 & 24 & 0.338 \\
\hline BMI & $22.65 \pm 4.07$ & $22.23 \pm 3.58$ & $22.48 \pm 3.87$ & 0.471 \\
\hline Length of hospital & $9.69 \pm 7.09$ & $9.47 \pm 4.12$ & $9.60 \pm 6.00$ & 0.696 \\
\hline Days between fracture and hospitalization (days) & $2.02 \pm 4.66$ & $1.44 \pm 3.14$ & $1.77 \pm 4.08$ & 0.115 \\
\hline Days between fracture and operation (days) & $6.49 \pm 5.45$ & $5.16 \pm 5.70$ & $5.93 \pm 5.58$ & 0.013 \\
\hline \multicolumn{5}{|l|}{ ASA classification } \\
\hline 1 & 11 & 17 & 28 & \multirow[t]{4}{*}{0.047} \\
\hline 2 & 182 & 113 & 295 & \\
\hline 3 & 70 & 66 & 136 & \\
\hline 4 & 2 & 2 & 4 & \\
\hline \multicolumn{5}{|l|}{ Surgical procedures } \\
\hline ORIF & 151 & 127 & 278 & \multirow[t]{4}{*}{0.099} \\
\hline Hemiarthroplasty & 93 & 63 & 156 & \\
\hline THR & 21 & 7 & 28 & \\
\hline $\mathrm{EF}$ & 0 & 1 & 1 & \\
\hline Duration of operation (mins) & $98.37 \pm 47.67$ & $92.42 \pm 48.81$ & $95.82 \pm 48.20$ & 0.191 \\
\hline Transfusion $(\mathrm{U})$ & $1.23 \pm 1.46$ & $1.10 \pm 1.37$ & $1.17 \pm 1.42$ & 0.335 \\
\hline Blood loss $(\mathrm{mL})$ & $275.55 \pm 163.49$ & $231.68 \pm 117.12$ & $256.82 \pm \mid 46.97$ & 0.001 \\
\hline Liquid transfusion $(\mathrm{mL})$ & 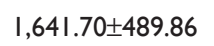 & $\mathrm{I}, 626.53 \pm 459.22$ & $\mathrm{I}, 635.24 \pm 476.60$ & 0.736 \\
\hline Drainage $(\mathrm{mL})$ & $|72.4| \pm 59.30$ & $157.67 \pm 68.98$ & $165.4 \mid \pm 64.29$ & 0.151 \\
\hline Post-operative infection & I & 0 & I & 1.000 \\
\hline \multicolumn{5}{|l|}{ Serum markers } \\
\hline D-dimer at admission (mg/L) & $15.39 \pm 16.34$ & $15.15 \pm 19.01$ & $15.29 \pm 17.54$ & 0.885 \\
\hline D-dimer preoperatively (mg/L) & $7.48 \pm 10.26$ & $4.94 \pm 4.65$ & $6.50 \pm 8.60$ & 0.016 \\
\hline D-dimer at postoperative I day (mg/L) & $10.88 \pm 10.00$ & $6.38 \pm 5.80$ & $8.96 \pm 8.74$ & 0.000 \\
\hline D-dimer at postoperative 5 day $(\mathrm{mg} / \mathrm{L})$ & $10.95 \pm 7.84$ & $6.48 \pm 3.90$ & $8.83 \pm 6.65$ & 0.000 \\
\hline CRP at admission $(\mathrm{mg} / \mathrm{L})$ & $20.25 \pm 31.83$ & $19.17 \pm 30.97$ & $19.75 \pm 31.39$ & 0.771 \\
\hline CRP at postoperative 5 day (mg/L) & $35.96 \pm 52.29$ & $21.36 \pm 36.11$ & $29.47 \pm 46.27$ & 0.025 \\
\hline
\end{tabular}

Abbreviations: ASA, American Society of Anesthesiologists; BMI, body mass index; CRP, C-reactive protein; EF, external fixation; ORIF, open reduction internal fixation; THR, total hip replacement.

and $87.17 \%$ of all preoperative and postoperative DVTs, respectively.

Too many factors contribute to the formation of thrombosis after trauma, ${ }^{14,15}$ including fracture and operation. ${ }^{5,16}$ In this study, we found that not only was the incidence of postoperative DVT higher than preoperative DVT but also that the incidence of DVT in the uninjured lower extremity increased from $13.60 \%$ to $25.05 \%$. Hypercoagulation state, a boosting thrombosis factor, may be the main contributing factor to this phenomenon. Selby et al and Meissner et al 


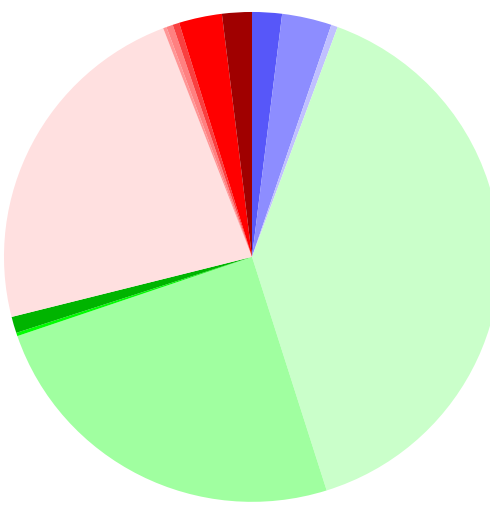

Figure 2 Dynamic changes of DVT before and after operation

Abbreviation: DVT, deep vein thrombosis.

proved that hypercoagulability state persists for a long time after fractures. ${ }^{7,17}$ In addition, the operation itself is the introducing factor of hypercoagulability states. ${ }^{18,19}$ Thus, the occurrence of DVT in the uninjured limb and increased incidence of DVT are easy to comprehend. Similar to operation, blood loss is another factor that promotes hypercoagulability states..$^{20}$ In the multivariate analysis of postoperative DVT, blood loss was an independent risk factor (OR, 1.002; $P=0.014)$. Blood loss in the thrombosis group $(275.55 \pm 163.49 \mathrm{~mL})$ was greater than the non-thrombosis group $(231.68 \pm 117.12 \mathrm{~mL})$. However, no significant differences in transfusion, liquid transfusion, and postoperative drainage were found. We think that the more the blood loss, the higher the level of coagulation, which is contributing to the thrombosis.

Furthermore, age, venous thrombosis at admission, and number of days between the fracture and the operation were the 3 important contributing factors to preoperative DVT. This result is similar to those of previous studies. ${ }^{12,21,22}$ In our hospital, which is a level I trauma center in our region, 66 patients with DVT at admission were referred from other trauma centers. Therefore, venous thrombosis at admission became a risk factor of preoperative and postoperative DVTs. What calls for special attention is that coronary heart disease is another important contributing factor to postoperative DVT. Zheng et al reported that prethrombotic state was an independent risk factor of perioperative events in patients with coronary heart disease ${ }^{23}$ and that the relationship between hypercoagulability states and coronary heart disease may be close. In additional, the number of days between the fracture and the operation was also important to thrombosis. There were 132 patients undergoing surgery within $72 \mathrm{~h}$ of fracture, the analysis shows earlier intervention could save

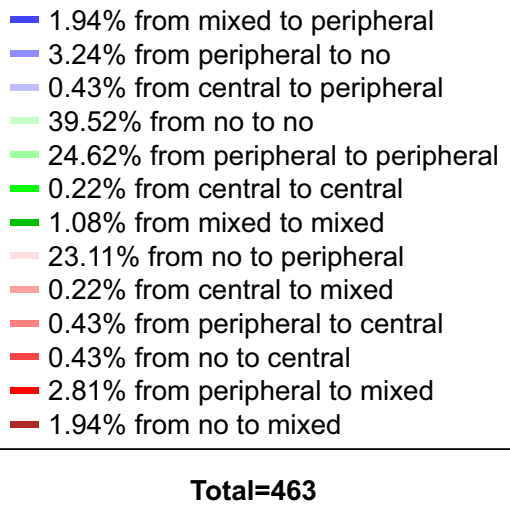

Total $=463$

$11.85 \%(127 / 331-35 / 132)$ in preoperative DVT $(P=0.016)$, but not in postoperative DVT. There were 198 patients undergoing surgery within $72 \mathrm{~h}$ of admission, earlier intervention could save $9.11 \%(162 / 265-103 / 198)$ in postoperative DVT $(P=0.050)$, but not in preoperative DVT.

The dynamic changes of DVT before and after operation were obvious, and the increase in the incidence of mainly postoperative DVT was high. Of 118 new postoperative DVTs, most were peripheral DVTs. Especially, peripheral DVTs are likely to occur in calf muscle veins. ${ }^{24}$ Of 303 patients who had no changes in hospitalization duration, $183 \mathrm{had}$ no DVT from preoperatively to postoperatively. The DVTs in 114 patients were peripheral from preoperatively to postoperatively. This result is similar to that reported by Song et al, ${ }^{5}$ in which most patients diagnosed as having DVT postoperatively already had DVT before the operation.

In this study, the prevalence of DVT in patients with hip fractures in the bilateral lower extremities was high, from $34.98 \%$ preoperatively to $57.23 \%$ postoperatively. The previous studies reported lower incidence rates than that in our study, ranging from $11.1 \%$ to $32.8 \%$ preoperatively or postoperatively. ${ }^{4-6}$ The reasons are varied. First, most studies that detected DVT were focused on the injured limb, ${ }^{4-6}$ limiting the data to those from uninjured limbs. The incidence reported by Mok was $14.3 \%,{ }^{9}$ with a preoperative incidence of $13.60 \%$ and a postoperative incidence of $25.05 \%$. Second, the mean number of days between the fracture and the operation was 5.93 days in our study. However, previous studies showed that the preoperative prevalence of DVT ranged from $54 \%$ to $62 \%$ in patients with acute fractures whose surgery was delayed by $>48 \mathrm{~h}^{25,26}$ Most of the patients in this study often presented a few days after the fracture, mainly because of transportation from other hospitals to our 
trauma center and the poor comorbidity of elderly patients, which needs more time for preoperative preparation. ${ }^{4,10}$ The delay in surgical intervention may predispose patients to developing thromboembolic problems. Thus, these factors may contribute to the higher prevalence in this study than in other studies.

\section{Conclusion}

The actual incidence of DVT after a hip fracture may be underestimated. The incidences of preoperative and postoperative DVT were high. The incidence of DVT in the uninjured limb was also high.

\section{Acknowledgment}

This study was supported by the Social Development Foundation of Shaanxi Province (grant number 2017ZDXMSF-009).

\section{Disclosure}

The authors have no funding or commercial associations (eg, consultancies, stock ownership, equity interest, patent/ licensing arrangements, etc.) that might pose a conflict of interest in connection with the submitted article. The authors report no conflicts of interest in this work.

\section{References}

1. Brill JB, Badiee J, Zander AL, et al. The rate of deep vein thrombosis doubles in trauma patients with hypercoagulable thromboelastography. J Trauma Acute Care Surg. 2017;83(3):413-419.

2. Di Nisio M, van Es N, Buller HR. Deep vein thrombosis and pulmonary embolism. Lancet. 2016;388(10063):3060-3073.

3. Testroote M, Stigter WA, Janssen L, et al. Low molecular weight heparin for prevention of venous thromboembolism in patients with lower-leg immobilization. Cochrane Database Syst Rev. 2014;(4):CD006681.

4. Shin WC, Woo SH, Lee SJ, Lee JS, Kim C, Suh KT. Preoperative prevalence of and risk factors for venous thromboembolism in patients with a hip fracture: an indirect multidetector CT venography study. J Bone Joint Surg Am. 2016;98(24):2089-2095.

5. Song K, Yao Y, Rong Z, Shen Y, Zheng M, Jiang Q. The preoperative incidence of deep vein thrombosis (DVT) and its correlation with postoperative DVT in patients undergoing elective surgery for femoral neck fractures. Arch Orthop Trauma Surg. 2016;136(10):1459-1464.

6. Tang Y, Wang K, Shi Z, Yang P, Dang X. A RCT study of Rivaroxaban, low-molecular-weight heparin, and sequential medication regimens for the prevention of venous thrombosis after internal fixation of hip fracture. Biomed Pharmacother. 2017;92:982-988.

7. Meissner MH, Chandler WL, Elliott JS. Venous thromboembolism in trauma: a local manifestation of systemic hypercoagulability? J Trauma. 2003;54(2):224-231.
8. Decker S, Weaver MJ. Deep venous thrombosis following different isolated lower extremity fractures: what is known about prevalences, locations, risk factors and prophylaxis? Eur J Trauma Emerg Surg. 2013; 39(6):591-598.

9. Mok CK, Hoaglund FT, Rogoff SM, Chow SP, Ma A, Yau AC. The incidence of deep vein thrombosis in Hong Kong Chinese after hip surgery for fracture of the proximal femur. Br J Surg. 1979;66(9):640-642.

10. Sun Y, Chen D, Xu Z, et al. Incidence of symptomatic and asymptomatic venous thromboembolism after elective knee arthroscopic surgery: a retrospective study with routinely applied venography. Arthroscopy. 2014;30(7):818-822.

11. Froehlich JA, Dorfman GS, Cronan JJ, Urbanek PJ, Herndon JH, Aaron RK. Compression ultrasonography for the detection of deep venous thrombosis in patients who have a fracture of the hip. A prospective study. J Bone Joint Surg Am. 1989;71(2):249-256.

12. Smith EB, Parvizi J, Purtill JJ. Delayed surgery for patients with femur and hip fractures-risk of deep venous thrombosis. J Trauma. 2011;70(6): E113-E116.

13. Rabinov K, Paulin S. Roentgen diagnosis of venous thrombosis in the leg. Arch Surg. 1972;104(2):134-144.

14. Chen F, Xiong JX, Zhou WM. Differences in limb, age and sex of Chinese deep vein thrombosis patients. Phlebology. 2015;30(4):242-824.

15. Park MS, Perkins SE, Spears GM, et al. Risk factors for venous thromboembolism after acute trauma: a population-based case-cohort study. Thromb Res. 2016;144:40-45.

16. Park SJ, Kim CK, Park YS, Lim SJ, Kim SM. Incidence and factors predicting venous thromboembolism after surgical treatment of fractures below the hip. J Orthop Trauma. 2015;29(10):e349-e354.

17. Selby R, Geerts W, Ofosu FA, et al. Hypercoagulability after trauma: hemostatic changes and relationship to venous thromboembolism. Thromb Res. 2009;124(3):281-287.

18. Kupcinskiene K, Trepenaitis D, Petereit R, et al. Monitoring of hypercoagulability by thromboelastography in bariatric surgery. Med Sci Monit. 2017;23:1819-1826.

19. Wilson D, Cooke EA, McNally MA, Wilson HK, Yeates A, Mollan RA. Changes in coagulability as measured by thrombelastography following surgery for proximal femoral fracture. Injury. 2001;32(10):765-770.

20. Riha GM, Kunio NR, Van PY, et al. Uncontrolled hemorrhagic shock results in a hypercoagulable state modulated by initial fluid resuscitation regimens. J Trauma Acute Care Surg. 2013;75(1):129-134.

21. Poh KS, Lingaraj K. Complications and their risk factors following hip fracture surgery. J Orthop Surg (Hong Kong). 2013;21(2):154-157.

22. Lee SY, Ro du H, Chung CY, et al. Incidence of deep vein thrombosis after major lower limb orthopedic surgery: analysis of a nationwide claim registry. Yonsei Med J. 2015;56(1):139-145.

23. Zheng H, Ma HP, Chen L, Zhan HT, Guo H. Prethrombotic state and cardiac events in patients with coronary heart disease during noncardiac surgery. Clin Appl Thromb Hemost. 2014;20(1):84-90.

24. Henry JC, Satiani B. Calf muscle venous thrombosis: a review of the clinical implications and therapy. Vasc Endovascular Surg. 2014; 48(5-6):396-401.

25. Hefley FG Jr, Nelson CL, Puskarich-May CL. Effect of delayed admission to the hospital on the preoperative prevalence of deep-vein thrombosis associated with fractures about the hip. J Bone Joint Surg Am. 1996; 78(4):581-583.

26. Zahn HR, Skinner JA, Porteous MJ. The preoperative prevalence of deep vein thrombosis in patients with femoral neck fractures and delayed operation. Injury. 1999;30(9):605-607. 


\section{Publish your work in this journal}

Clinical Interventions in Aging is an international, peer-reviewed journal focusing on evidence-based reports on the value or lack thereof of treatments intended to prevent or delay the onset of maladaptive correlates of aging in human beings. This journal is indexed on PubMed Central, MedLine,

\section{Dovepress}

CAS, Scopus and the Elsevier Bibliographic databases. The manuscript management system is completely online and includes a very quick and fair peer-review system, which is all easy to use. Visit http://www.dovepress. $\mathrm{com} /$ testimonials.php to read real quotes from published authors. 